TRANSACTIONS OF THE

AMERICAN MATHEMATICAL SOCIETY

Volume 353, Number 9, Pages 3585-3602

S 0002-9947(01)02802-1

Article electronically published on April 26, 2001

\title{
HAUSDORFF CONVERGENCE AND UNIVERSAL COVERS
}

\author{
CHRISTINA SORMANI AND GUOFANG WEI
}

\begin{abstract}
We prove that if $Y$ is the Gromov-Hausdorff limit of a sequence of compact manifolds, $M_{i}^{n}$, with a uniform lower bound on Ricci curvature and a uniform upper bound on diameter, then $Y$ has a universal cover. We then show that, for $i$ sufficiently large, the fundamental group of $M_{i}$ has a surjective homeomorphism onto the group of deck transforms of $Y$. Finally, in the non-collapsed case where the $M_{i}$ have an additional uniform lower bound on volume, we prove that the kernels of these surjective maps are finite with a uniform bound on their cardinality. A number of theorems are also proven concerning the limits of covering spaces and their deck transforms when the $M_{i}$ are only assumed to be compact length spaces with a uniform upper bound on diameter.
\end{abstract}

\section{INTRODUCTION}

In recent years the limit spaces of manifolds with lower bounds on Ricci curvature have been studied from both a geometric and topological perspective. In particular, Cheeger and Colding have proven a number of results regarding the regularity and geometric properties of these spaces. However, the topology of the limit spaces is less well understood. Note that in this paper a manifold is a Riemannian manifold without boundary.

Anderson $[\mathrm{An}]$ has proven that there are only finitely many isomorphism types of fundamental groups of manifolds with a uniform upper bound on diameter, lower bound on volume and lower bound on Ricci curvature. Thus one might think that given a converging sequence of such manifolds, the fundamental groups of the manifolds must eventually be isomorphic to the fundamental group of the limit space. However, Otsu $\mathrm{Ot}$ has shown that there are metrics of uniformly positive Ricci curvature on $S^{3} \times \mathbb{R} P^{2}$ which converge to a simply connected 5-dim metric space, showing that this need not be the case.

Tuschmann $[\mathrm{Tu}]$ has proven that if $Y$ is the limit space of a sequence of manifolds with two sided sectional curvature bounds then $Y$ is locally simply connected and thus has a universal cover [Defn. 2.3]. In fact Perelman [Pl] shows that the limit space of a sequence of manifolds with a lower bound on sectional curvature is locally contractible. If the limit space is locally simply connected, it is not difficult to show that eventually there is a surjective map from the fundamental groups of the manifolds onto the fundamental group of the limit space (see $[\mathrm{Tu}] \mathrm{Ca}], \mathrm{Gr}$, page 100], also Section 2 of this paper). Zhu [Zh] has proven a similar result for limits

Received by the editors September 6, 2000.

1991 Mathematics Subject Classification. Primary 53C20.

Partially supported by NSF Grant \#DMS-9971833. 
of three dimensional manifolds with uniform lower bounds on Ricci curvature and volume and a uniform upper bound on diameter.

In this paper a manifold is a Riemannian manifold without boundary. Here we are concerned with limits of sequences of manifolds with a uniform upper bound on diameter and lower bound on Ricci curvature. The limits of such sequences have only been shown to be locally simply connected at special "regular" points $\mathrm{ChCo}$. In fact Menguy [Me] has shown that the limit space could locally have infinite topological type.

We prove that the universal cover of the limit space exists [Defn. 2.3. We can thus study the group of deck transforms of the universal cover, $\bar{\pi}_{1}(M)$ [Defn. 2.4]. Note that this revised fundamental group, $\bar{\pi}_{1}(M)$, is isomorphic to the fundamental group of $M$ if $M$ is locally simply connected (cf. [Sp]). We can now state the main theorem of our paper.

Theorem 1.1. Let $M_{i}$ be a sequence of compact manifolds satisfying

$$
\operatorname{Ricci}\left(M_{i}\right) \geq(n-1) H \text { and } \operatorname{diam}\left(M_{i}\right) \leq D,
$$

for some $H \in \mathbb{R}$ and $D>0$. If $Y$ is the Gromov-Hausdorff limit of the $M_{i}$ then the universal cover of $Y$ exists and for $N$ sufficiently large depending on $Y$, there is a surjective homomorphism

$$
\Phi_{i}: \pi_{1}\left(M_{i}\right) \rightarrow \bar{\pi}_{1}(Y) \quad \forall i \geq N .
$$

Note 1.2. There are Hausdorff limits of compact manifolds with a uniform bound on diameter which have no universal cover (see Example 2.7).

Note 1.3. In Theorem 1.1, we do not know whether $\tilde{Y}$ is simply connected or not. Example 2.6 demonstrates that without the curvature condition, it need not be simply connected.

When the sequence is non-collapsing we have a stronger result:

Theorem 1.4. Let $M_{i}^{n}$ be a sequence of compact manifolds satisfying

$$
\operatorname{Ricci}\left(M_{i}\right) \geq(n-1) H, \quad \operatorname{diam}\left(M_{i}\right) \leq D \quad \text { and } \operatorname{vol}\left(M_{i}\right) \geq V
$$

for some $H \in \mathbb{R}, D>0$ and $V>0$. If $Y$ is the Gromov-Hausdorff limit of the $M_{i}$ then there is $i_{0}=i_{0}\left(n, H, V, D, \delta_{Y}\right)$ such that $\pi_{1}\left(M_{i}\right) / F_{i}$ is isomorphic to $\bar{\pi}_{1}(Y)$ for all $i \geq i_{0}$, here $F_{i}$ is a finite subgroup of $\pi_{1}\left(M_{i}\right)$, and the order of each $F_{i}$ is uniformly bounded by $N(n, H, V, D)$. In particular, $\pi_{1}\left(M_{i}\right) / F_{i}$ is isomorphic to $\pi_{1}\left(M_{j}\right) / F_{j}$ for all $i, j \geq i_{0}$.

Compare Anderson's result An which says that there are only finitely many isomorphism types of fundamental groups of compact manifolds satisfying (1.3).

To prove these results we need to study the limit spaces of compact length spaces. Thus in Sections 2 and 3 we restrict ourselves to sequences, $M_{i}$, which are only compact length spaces with $\operatorname{diam}\left(M_{i}\right) \leq D$ that converge in the Gromov-Hausdorff sense to a limit space $Y$.

In Section 2 we present two examples of such sequences of length spaces which converge in the Gromov-Hausdorff topology. However, their fundamental groups cannot be mapped surjectively onto the fundamental group or revised fundamental group of the limit space. In the first example, we have a sequence of simply connected length spaces whose limit space is not simply connected [Ex. 2.6]. In the second example, the limit space has no universal cover [Ex. [2.7]. It should be 
recalled that even when the limit space is a manifold that the limit of the universal covers is not necessarily a cover of the limit space (see [Pe1 Theorem 2.1] for a case where it is). Thus the universal cover cannot be directly used to prove properties about the fundamental group.

In Section 3, we introduce $\delta$-covering spaces [Defn. 3.1]. Unlike the universal cover, $\delta$-covers always exist. We then show that the limit of the $\delta$-covers is a cover of the limit space [Theorem 3.6]. Furthermore, we prove that for a fixed $\delta>0$ group of deck transforms of the $\delta$-covers, $\tilde{M}_{i}^{\delta}$, of the $M_{i}$ eventually have a surjective map onto deck transforms of the $\delta$-cover of the limit space [Cor. 3.5]. We also describe the relationship with the $\delta$-covers and the universal cover if the latter exists [Theorem 3.7]. We conclude with a proof of the following theorem which should be contrasted with Example 2.6 [Note 2.8].

Theorem 1.5. If the $M_{i}$ are simply connected compact length spaces with diam $\left(M_{i}\right)$ $\leq D$ that converge in the Gromov Hausdorff topology to a compact space $Y$, then the universal cover of $Y$ exists and the revised fundamental group, $\bar{\pi}_{1}(Y)$, is trivial.

In Section 4 we study limit spaces, $Y$, of sequences of compact manifolds $M_{i}$ satisfying (1.1) and prove that the universal cover of $Y$ exists. To do so we first prove Lemma 4.6 by extending techniques from [So] involving the Excess Theorem of AbGl] to arbitrary lower bounds on Ricci curvature. We then apply this lemma combined with $\mathrm{ChCo}$ regularity results to prove Theorem 4.5 that there is at least one ball in the limit space that lifts isometrically to any covering space. To extend this to balls around arbitrary points in the limit space we use the Bishop-Gromov Volume Comparison, which holds for limit measures by $\mathrm{ChCO}$ and Theorem 3.6 Thus we prove that there is a $\delta_{Y}>0$ such that the $\delta_{Y}$-cover of the limit space $Y$ is the universal cover of $Y$ [Theorem 4.8]. Note that this $\delta_{Y}$ depends on many factors and cannot be determined uniformly without contradicting the examples of Otsu Ot. Combining Theorem 4.8 with the results of Section 3, we obtain Theorem 1.1 and Corollary 4.12 .

We conclude with the non-collapsed case, where $M_{i}$ satisfy (1.3), proving Theorem [1.4 We use results from Section 3 regarding the kernel of the surjective map and results of Anderson regarding elements of the fundamental group of manifolds with lower bounds on volume $\mathrm{An}$ ] to obtain Theorem 1.4.

Background material for Gromov-Hausdorff limits and Ricci curvature can be found in Chapter 1 Sections A-C, Chapter 3 Sections A-B, Chapter 5 Section A of $\mathrm{Gr}$ and in Chapters 9-10 of $\mathrm{Pe} 2$. Background material on covering spaces and fundamental groups can be found in Chapters 1-2 of [Sp] and [Ma].

\section{Background And ExAmples}

In Sections 2 and 3 we consider compact length spaces. No curvature condition is assumed. See Gr, Chapter 1] for basic results about length spaces (called path metric spaces). Recall also (cf. [Gr, Chapter 3A]), the following definition of the Gromov-Hausdorff distance between metric spaces (called Hausdorff distance).

Definition 2.1 ( $\mathrm{Gr}$, Defn. 3.4]). Given two metric spaces $X$ and $Y$, the GromovHausdorff distance between them is defined,

$$
d_{G H}(X, Y)=\inf \left\{d_{H}^{Z}(f(X), f(Y)): \begin{array}{l}
\text { for all metric spaces } Z, \text { and isometric } \\
\text { embeddings } f: X \rightarrow Z, g: Y \rightarrow Z
\end{array}\right\}
$$


where, $d_{H}^{Z}$ is the Hausdorff distance between subsets of $Z$,

$$
d_{H}^{Z}(A, B)=\inf \left\{\epsilon>0: B \subset T_{\epsilon}(A) \text { and } A \subset T_{\epsilon}(B)\right\} .
$$

Here $T_{\epsilon}(A)=\left\{x \in Z: d_{Z}(x, A)<\epsilon\right\}$.

If $x \in X$ and $y \in Y$, the pointed Gromov-Hausdorff distance:

$$
d_{G H}((X, x),(Y, y))=\inf \left\{\begin{array}{l}
d_{H}^{Z}(f(X), f(Y)): \\
\text { for all metric spaces } Z, \text { and isometric embed- } \\
\text { dings } f: X \rightarrow Z, g: Y \rightarrow Z \text { s.t. } f(x)=g(y)
\end{array}\right\}
$$

It is then clear what we mean by the Gromov-Hausdorff convergence of compact metric spaces. However, for non-compact metric spaces, the following looser definition of convergence is used.

Definition 2.2 ( $\mathrm{Gr}$, Defn. 3.14]). We say that non-compact length spaces $\left(X_{n}, x_{n}\right)$ converge in the Gromov-Hausdorff sense to $(Y, y)$ if for any $R>0$ there exists a sequence $\epsilon_{n} \rightarrow 0$ such that $B_{x_{n}}\left(R+\epsilon_{n}\right)$ converges to $B_{y}(R)$ in the Gromov-Hausdorff sense.

Although we are limiting ourselves to compact length spaces, their universal covers may well be non-compact.

Recall that a space, $Y$, is semi-locally simply connected (or semi-locally one connected) if for all $y \in Y$ there is a neighborhood $U$ of $y$ such that $\pi_{1}(U, y) \rightarrow$ $\pi_{1}(Y, y)$ is trivial $([\mathrm{Sp}$ p. 78$]$ [Ma, p. 142]). That is, any curve in $U$ is contractible in $Y$. This is weaker than saying that $U$ is simply connected.

For a metric space $Y$, let $r(Y)$ denote the maximal number $r$ such that every closed curve in a ball of radius $r$ in $Y$ is homotopic to zero in $Y$, the semi-locally simply connectivity radius. Note that if $Y$ is compact and semi-locally simply connected, then $r(Y)$ is positive.

The following theorem demonstrates how Gromov-Hausdorff closeness affects the fundamental groups of spaces which are semi-locally simply connected.

Theorem 2.1. Let $Y_{1}, Y_{2}$ be two compact length spaces and $Y_{2}$ semi-locally simply connected. If $d_{G H}\left(Y_{1}, Y_{2}\right) \leq \epsilon$, where $\epsilon=r\left(Y_{2}\right) / 20$, then there exists a surjective homomorphism $\Phi: \pi_{1}\left(Y_{1}\right) \rightarrow \pi_{1}\left(Y_{2}\right)$.

This theorem essentially follows from the proof of [Tu, Theorem (b)]. See also [Gr, page 100] and [Ca. As an extension of this theorem, Theorem 3.4, will be proven in the next section; we will omit the proof.

Note 2.2. One usually doesn't get a similar surjection for high homotopy groups, as Berger's examples of $S^{3}$ collapsing to $S^{2}$ illustrate for $\pi_{2}$.

Theorem 2.1 immediately gives the following isomorphism.

Corollary 2.3. Let $Y_{1}$ and $Y_{2}$ be two compact semi-locally simply connected length spaces and let $r=\min \left\{r\left(Y_{1}\right) / 20, r\left(Y_{2}\right) / 20\right\}$. If $d_{G H}\left(Y_{1}, Y_{2}\right) \leq r$, then $\pi_{1}\left(Y_{1}\right)$ is isomorphic to $\pi_{1}\left(Y_{2}\right)$.

Note 2.4. Note that if $Y_{1}=S^{2} \times S_{\epsilon}^{1}$ and $Y_{2}=S^{2}$, then $\pi_{1}\left(Y_{1}\right)$ only maps surjectively onto $\pi_{1}\left(Y_{2}\right)$. This does not contradict the above corollary because $r$ there depends on both $r\left(Y_{1}\right)=\epsilon$ and $r\left(Y_{2}\right)$. 
Note 2.5. Note that Colding $\left[\mathrm{Co}\right.$ and Cheeger-Colding $[\mathrm{ChCo}]$, proved that if $Y_{1}, Y_{2}$ are the same dimensional manifolds and sufficiently Hausdorff close (closeness depends on $\left.Y_{1}, Y_{2}\right)$, then $Y_{1}, Y_{2}$ are diffeomorphic. Here an assumption on $r\left(Y_{i}\right)$ is shown to suffice to match the fundamental groups of length spaces.

Definition 2.3 ([Sp, pp. 62, 82]). $Y$ is a covering space of $X$ if there is a continuous map $\pi: Y \rightarrow X$ such that $\forall x \in X$ there is an open neighborhood $U$ such that $\pi^{-1}(U)$ is a disjoint union of open subsets of $Y$ each of which is mapped homeomorphically onto $U$ by $\pi$. We say that a connected space $\tilde{X}$ is a universal cover of $X$ if $\tilde{X}$ is a cover of $X$ such that for any other cover $Y$ of $X$, there is a commutative triangle formed by a continuous map $f: \tilde{X} \rightarrow Y$ and the two covering projections.

Recall that if $\pi: E \rightarrow Y$ is a covering and $Y$ is a connected compact length space, then there is a unique length metric on $E$ making $\pi: E \rightarrow Y$ distance nonincreasing and a local isometry (see $[\mathrm{Ri}]$ ). Of course, the covering space need not be compact.

The universal cover may not exist as can be seen in [Sp, Ex. 17, p. 84]. However, if it exists, then it is unique. Furthermore, if a space is locally path connected and semi-locally simply connected, then it has a universal cover and that cover is simply connected [Sp, Cor. 14, p. 83]. On the other hand, the universal covering space of a locally path connected space may not be simply connected [Sp, Ex. 18, p. 84]. We now present two examples.

Example 2.6. There exists a compact length space, $X$, which is the limit of simply connected compact length spaces, but is not simply connected.

Proof. The construction of $X$ is based upon the fact that $\{(t, \sin (1 / t)): 0<t \leq$ $1 / \pi\} \cup\{0\} \times[-1,1]$ is a compact metric space which isn't path connected. Here, however, we construct a path connected space which isn't simply connected.

First define two compact sets:

$$
\begin{gathered}
K_{1}=\{(x, y, \sin (1 / y)): y \in(0,1 / \pi],|x| \leq y\}, \\
K_{2}=\{(x, y, z):|x|=y \leq 1 / \pi, z \in[-2,2]\} .
\end{gathered}
$$

Let the length space be

$$
X=K_{1} \cup K_{2} .
$$

First we prove that $X$ is not simply connected.

We claim that the loops $C_{j}:[0,4 /(j \pi)] \rightarrow X$ defined as follows are all homotopic to each other but are not contractible.

$$
\begin{gathered}
C_{j}(t)=(-t, t, 0) \subset K_{2}, \quad t \in[0,1 /(j \pi)], \\
C_{j}(t)=(t-2 /(j \pi), 1 /(j \pi), 0) \subset K_{1}, \quad t \in[1 /(j \pi), 3 /(j \pi)], \\
C_{j}(t)=(4 /(j \pi)-t, 4 /(j \pi)-t, 0), \quad t \in[3 /(j \pi), 4 /(j \pi)] .
\end{gathered}
$$

Clearly the length of this curve is

$$
L\left(C_{j}\right)=(2 \sqrt{2}+2) /(j \pi) \rightarrow 0, \quad j \rightarrow \infty,
$$

and clearly each curve is homotopic to the next. 
Now why aren't they contractible? If $C_{1}$ were contractable, there would be a homotopy

$$
H: A=[-1 / \pi, 1 / \pi] \times[0,1 / \pi] \rightarrow X \subset R^{3}
$$

such that

$$
\begin{aligned}
& H(t, 0)=(0,0,0), \quad H(-1 / \pi, s)=(-s, s, 0), \\
& H(1 / \pi, s)=(s, s, 0), \quad H(t, 1 / \pi)=(t, 1 / \pi, 0) .
\end{aligned}
$$

Write $H(s, t)=\left(h_{1}(s, t), h_{2}(s, t), h_{3}(s, t)\right)$.

It is easy to see that $X_{r}=X-K_{1} \cap\{(x, y, z): z>r\}$ is not simply connected for $r=3 / 4$, and that $C_{1}$ is not contractible in this set. Thus $H^{-1}\left(X_{3 / 4}\right)$ is also not simply connected and thus cannot contain the entire domain. So there exists $\left(s_{1}, t_{1}\right) \in A$ such that $h_{3}\left(s_{1}, t_{1}\right)>3 / 4$ and $H\left(s_{1}, t_{1}\right) \in K_{1}$.

Let $U_{1} \subset A$ be the connected component of $H^{-1}\left(X \backslash X_{1 / 2}\right)$ that contains $\left(s_{1}, t_{1}\right)$.

We continue this definition inductively, given $\left(s_{j}, t_{j}\right)$ we define $U_{j} \subset A$ to be the connected component of $H^{-1}\left(X \backslash X_{1 / 2}\right)$ that contains $\left(s_{j}, t_{j}\right)$. Then we note that $C_{1}$ is not contractable in $H^{-1}\left(X_{3 / 4} \cup H\left(U_{1}\right) \cup \ldots \cup H\left(U_{j}\right)\right)$ because at most finitely many peaks have been filled in. So there exists $\left(s_{j+1}, t_{j+1}\right) \in A \backslash\left(U_{1} \cup \ldots \cup U_{j}\right)$ such that $h_{3}\left(s_{j+1}, t_{j+1}\right)>3 / 4$ and $H\left(s_{j+1}, t_{j+1}\right) \in K_{1}$.

Now $A$ is compact so a subsequence of the $\left(s_{j}, t_{j}\right)$ converges to some $\left(s_{\infty}, t_{\infty}\right) \in$ $A$. By continuity $h_{3}\left(s_{\infty}, t_{\infty}\right) \geq 3 / 4$. Now each point in the subsequence is in a distinct connected component of $H^{-1}\left(X \backslash X_{1 / 2}\right)$. Thus there exist points in $H^{-1}\left(X_{1 / 2}\right)$ on line segments between the points in the subsequence. The limit of these points must have $h_{3} \leq 1 / 2$. Since these line segments are shorter and shorter, the limit point of points on the segments must also be $\left(s_{\infty}, t_{\infty}\right)$ and we have a contradiction. No homotopy can exist.

Now we define $X_{k}$, a sequence of simply connected length spaces which converge to $X$.

$$
\begin{gathered}
K_{k, 1}=\{(x, y, \sin (1 / y)): 1 /(k \pi) \leq y \leq 1 / \pi,|x| \leq y\} \\
K_{k, 1}^{\prime}=\{(x, y, 0): 0 \leq y \leq 1 /(k \pi),|x| \leq y\}
\end{gathered}
$$

and the connecting set

$$
K_{k, 2}=\{(x, y, z):|x|=y \leq 1 / \pi, z \in[-2,2]\} .
$$

Then the length spaces are defined,

$$
X_{k}=K_{k, 1} \cup K_{k, 1}^{\prime} \cup K_{k, 2} .
$$

It is easy to see that these spaces are compact with a uniform bound on their diameters. They are also easily seen to be simply connected.

Now we claim $d_{G H}\left(X_{k}, X\right)<10 /(k \pi)$. Let $Z_{k}$ be a metric space defined as the disjoint union of $X_{k}$ and $X$ with $K_{2}$ identified with $K_{k, 2}$ and $K_{k, 1}$ identified with the appropriate part of $K_{1}$. It is easy to see that the tubular neighborhood of radius $10 /(k \pi)$ about $X_{k}$ in $Z_{k}$ contains $X$ and vice versa because the tubular neighborhood about $K_{2}$ covers $Z_{k} \backslash\left(X \cup X_{k}\right)$.

Example 2.7. There exists a compact length space, $X$, with no universal cover which is the limit of a sequence of manifolds, $X_{i}$. Furthermore, the fundamental groups of the $X_{i}$ are finitely generated but the fundamental group of the limit space is not. 
Proof. Let $X$ be the Hawaii Ring. That is $X \subset \mathbf{R}^{2}$ is defined

$$
X=\bigcup_{j=1}^{\infty} C_{j}
$$

where $C_{j}$ is the circle of radius $1 / 2^{j}$ around $\left(1 / 2^{j}, 0\right)$.

Let $Y_{i}=\bigcup_{j=1}^{i} C_{j} \subset \mathbf{R}^{2}$. Then $Y_{i}$ are compact length spaces which converge to $X$.

To construct the manifolds, $X_{i}$ we consider the tubular neighborhoods,

$$
T_{1 /\left(1002^{i}\right)}\left(Y_{i} \times\{0\}\right) \subset \mathbf{R}^{3} .
$$

The boundaries of these neighborhoods are topological manifolds and length spaces, and are within a Gromov-Hausdorff distance $2 \pi /\left(102^{i}\right)$ of the $Y_{i}$. They may be smoothed to create the manifolds $X_{i}$, which will then also converge to the length space $X$.

If a space has a universal cover, then we can define a revised fundamental group. First recall the definition of the group of deck transforms (or self-equivalences) of a cover $\pi: Y \rightarrow X$. This is the group of homeomorphisms $h: Y \rightarrow Y$ such that $\pi \circ h=\pi$ [Sp, p. 85]. It is denoted $G(Y, X)$.

Given $\tilde{p} \in Y$, there is a natural surjection $\Psi_{\tilde{p}}$ from the fundamental group, $\pi_{1}(X, p)$ to $G(Y, X)$ defined as follows. Note that given $g \in \pi_{1}(M, p)$ and $\tilde{p}$, it then has a representative loop, $C$. We can lift $C$ to a curve based at $\tilde{p}$ in the cover. This defines an action of $g$ on $\pi^{-1}(p) \subset \tilde{M}$ which can be extended uniquely to a deck transform of $M$. This map is surjective when $Y$ is path connected because given any $h \in G(Y, X)$ we can join $\tilde{p}$ to $h(\tilde{p})$ by a curve and then the curve's projection to the base space gives an element of $\pi_{1}(X, p)$. The kernel, $H_{p}$, consists of elements of the fundamental group $\pi_{1}(M, p)$, whose representative loops are still closed when they are lifted to the cover.

Note that when the universal cover is simply connected and locally path connected, then the group of deck transforms of the universal cover is isomorphic to the fundamental group of $X$ Sp, page 87, Cor. 4].

Definition 2.4. Let the revised fundamental group of $M$, denoted $\bar{\pi}_{1}(M)$, be the group of deck transforms of the universal cover of $M$.

Then $\bar{\pi}_{1}(M)=\pi_{1}(M, p) / H_{p}$ where $H_{p}$ is a normal subgroup described above. It is isomorphic to $\pi_{1}(M, p)$ when $M$ is semi-locally simply connected. In fact $H_{p}$ consists of those elements of $\pi_{1}(M, p)$ whose representative loops lift to closed curves in the universal cover. Thus if $\bar{\pi}_{1}(M, p)=\pi_{1}(M, p)$ then $H_{p}$ is trivial, so every loop in $M$ which lifts to a closed curve in $\tilde{M}$ is contractible. In particular, each loop in $\tilde{M}$ must project to a closed loop in $M$ which is contractible, so it must be contractible. Thus $\bar{\pi}_{1}(M)=\pi_{1}(M, p)$ iff the universal cover of $M$ is simply connected.

Note 2.8. In Example 2.6, we have a sequence of simply connected spaces which converge to a space which is not simply connected. However, the limit space is its own universal cover. Thus its revised fundamental group is trivial. In Section 3 we will prove that this is always the case [Theorem 1.5$]$. 


\section{3. $\delta$-Covering Spaces}

In this section we continue to study locally path connected length spaces. We would like to understand what happens to the fundamental groups of such spaces when we take their limits. Since we do not assume that they are semi-locally simply connected, we do not know if these spaces have simply connected universal covers. In fact they might not have universal covers at all. To circumvent this problem we introduce intermediate $\delta$-covering spaces.

Let $\mathcal{U}$ be any open covering of $Y$. For any $p \in Y$, by Sp page 81], there is a covering space, $\tilde{Y}_{\mathcal{U}}$, of $Y$ with covering group $\pi_{1}(Y, \mathcal{U}, p)$, where $\pi_{1}(Y, \mathcal{U}, p)$ is a normal subgroup of $\pi_{1}(Y, p)$, generated by homotopy classes of closed paths having a representative of the form $\alpha^{-1} \circ \beta \circ \alpha$, where $\beta$ is a closed path lying in some element of $\mathcal{U}$ and $\alpha$ is a path from $p$ to $\beta(0)$.

Definition 3.1. Given $\delta>0$, the $\delta$-cover, denoted $\tilde{Y}^{\delta}$, of a length space $Y$, is defined to be $\tilde{Y}_{\mathcal{U}_{\delta}}$ where $\mathcal{U}_{\delta}$ is the open covering of $Y$ consisting of all balls of radius $\delta$.

The covering group will be denoted $\pi_{1}(Y, \delta, p) \subset \pi_{1}(Y, p)$ and the group of deck transforms of $\tilde{Y}^{\delta}$ will be denoted $G(Y, \delta)=\pi_{1}(Y, p) / \pi_{1}(Y, \delta, p)$.

Note that $\tilde{Y}^{\delta_{1}}$ covers $\tilde{Y}^{\delta_{2}}$ when $\delta_{1} \leq \delta_{2}$.

Note also that $G(Y, \delta)$, denoted $G\left(\tilde{Y}^{\delta}, Y\right)$ in [Sp p. 86, Cor. 3], does not depend on $p$. One can think of $G(Y, \delta)$ as roughly corresponding to the long loops, of length at least $\delta$, in $\pi_{1}(Y, p)$.

There is a natural surjective map from $\pi_{1}(Y, p)$ to $G(Y, \delta)$, which depends on $\tilde{p} \in \tilde{Y}^{\delta}$, that we can call $\Psi_{\tilde{p}, \delta}$. (See the paragraph after Example2.7 in Section 2.)

Definition 3.2. When $Y$ is compact, then for any $h \in G(Y, \delta)$, we can define the (translative) $\delta$-length of $h$,

$$
l(h, \delta)=\min _{q \in \tilde{Y}^{\delta}} d_{\tilde{Y}^{\delta}}(q, h(q)) .
$$

For any $g \in \pi_{1}(Y, p)$

$$
l(g, \delta)=\min _{q \in \tilde{Y}^{\delta}} d_{\tilde{Y}^{\delta}}\left(q, \Psi_{\tilde{p}, \delta}(g)(q)\right) .
$$

Note that since this is a compact length space, there is a point $q \in \tilde{Y}$ and a curve $C$ from $q$ to $\Psi_{\delta}(g)(q)$ ) whose length is $l(g, \delta)$. Consequently there is a point $\pi(q) \in Y$ and a loop $\pi(C)$ based at the point, $q$, of length $l(g, \delta)$. Furthermore there is some path, $\alpha$, from $p$ to $\pi(q)$ such that $\alpha^{-1}(\pi(C)) \alpha$ is a representative curve for $g \in \pi_{1}(Y, p)$.

We have the following basic properties for $\delta$-length.

Lemma 3.1. For all nontrivial $g \in G(Y, \delta, p)$, the $\delta$-length of $g$,

$$
l(g, \delta) \geq \delta
$$

For all $\delta_{1} \leq \delta_{2}$ we have

$$
l\left(g, \delta_{1}\right) \geq l\left(g, \delta_{2}\right) .
$$

Proof. Suppose there exists a $g$ such that (3.9) fails to hold. Since $Y$ is a length space, there is a curve $\beta$ of length less than $\delta$ running from some point, $q \in \tilde{Y}^{\delta}$ to 
$g(q)$. There is also a curve $\alpha$ running from $\tilde{p}$ to $q$. Then $\pi(\alpha)$ runs $p$ to $\pi(q)$, and $\pi(\beta)$ is a loop in $B_{\pi(q)}(\delta)$, so

$$
g=\left[\pi(\alpha)^{-1} \circ \pi(\beta) \circ \pi(\alpha)\right] \in G(Y, \delta, p)
$$

is trivial by the definition.

As mentioned above, for all $\delta_{1} \leq \delta_{2}$, there exists $\alpha$ and $\beta$ in $\tilde{Y}^{\delta_{1}}$ such that $L(\beta)=l\left(g, \delta_{1}\right)$ and

$$
g=\left[\pi(\alpha)^{-1} \circ \pi(\beta) \circ \pi(\alpha)\right] \in \pi(Y, p) .
$$

Now $\tilde{Y}^{\delta_{1}}$ is the $\delta_{1}$-cover of $\tilde{Y}^{\delta_{2}}$, so $\alpha$ and $\beta$ can be projected to paths in $\tilde{Y}^{\delta_{1}}$. Thus $l\left(g, \delta_{1}\right) \geq l\left(g, \delta_{2}\right)$.

Proposition 3.2. If $Y$ is a compact length space that has a universal cover, then the universal cover is a $\delta$-cover, for some $\delta_{0}>0$. Thus $\tilde{Y}=\tilde{Y}^{\delta_{0}}=\tilde{Y}^{\delta}$ for all $\delta<\delta_{0}$.

Proof. If $Y$ has a universal cover, then for all $y \in Y$, there is an $r_{y}>0$ such that $B_{y}\left(r_{y}\right)$ is lifted isometrically to the universal cover. Suppose the universal cover is not a $\delta$-cover for any $\delta>0$. Then there exists $p_{i} \in Y$ and $C_{i}$ a loop based at $p_{i}$ in $B_{p_{i}}(1 / i)$ which lift non-trivially to the universal cover. $Y$ is compact so a subsequence of $p_{i}$ converges to $p_{\infty}$, and eventually some $C_{i}$ will be contained in $B_{p_{\infty}}\left(r_{p_{\infty}}\right)$ which is a contradiction.

Proposition 3.3. If $Y$ is a compact length space such that the universal cover exists and all nontrivial elements of $\pi_{1}(Y, p)$ have a positive $\delta$-length for some $\delta$, then $Y$ is semi-locally simply connected.

Proof. Let $\delta_{0}$ be defined as in Proposition 3.2 If $Y$ is not semi-locally simply connected, then there is a curve $C$ contained in some $B_{q}\left(\delta_{0}\right) \subset Y$ which is not contractible in $Y$. Let $\beta$ run from $p$ to $q$ and $g=\left[\beta \circ C \circ \beta^{-1}\right] \in \pi_{1}(Y, p)$. Note that $\Psi_{\delta_{0}}(g)$ is trivial and since $\tilde{Y}^{\delta}=\tilde{Y}^{\delta_{0}}$ for all $\delta<\delta_{0}$ by our choice of $\delta_{0}, \Psi_{\delta_{0}}(g)$ is trivial for all $\delta<\delta_{0}$. This contradicts the hypothesis.

Note that in the example in Section 2, there is a nontrivial element of the fundamental group with $0 \delta$-length for all $\delta$.

Now we have good covering spaces for $Y$, but we don't know if Bishop-Gromov volume comparison holds on these covers even if it does on $Y$. In order to find a good covering space such that Bishop-Gromov volume comparison also holds, we relate these covering to the covering from the sequence. Note that in general, the universal covering of sequences doesn't converge to the universal cover of the limit, e.g. the lens spaces, $S^{3} / Z_{p}$, converge to $S^{2}$. (See [Pe1, Theorem 2.1] for a case where it does.) However, we will show that this is almost true in the $\delta$-cover level (see Theorem [3.6). To prove this first we need a revised version of Theorem 2.1] which does not require a bound on the semi-locally simply connectivity radius.

Theorem 3.4. Let $Y_{1}, Y_{2}$ be two compact length spaces such that $d_{G H}\left(Y_{1}, Y_{2}\right) \leq \epsilon$, then there is a surjective homomorphism, $\Phi: G\left(Y_{1}, \delta_{1}\right) \rightarrow G\left(Y_{2}, \delta_{2}\right)$ for any $\delta_{1}>$ $20 \epsilon$ and $\delta_{2}>\delta_{1}+10 \epsilon$.

In the proof of this theorem and subsequent theorems, we think of $G(Y, \delta)=$ $\pi_{1}(Y, p) / \pi_{1}(Y, \delta, p)$ as $G(Y, \delta, p)$ consisting of equivalence classes of loops based at $p$ such that $C_{1} \sim C_{2}$ iff $C_{1} \circ C_{2}^{-1}$ is homotopic to a curve in $\pi_{1}(Y, \delta, p)$. 
Proof. Since $d_{G H}\left(Y_{1}, Y_{2}\right) \leq \epsilon$, there must exist a metric space $(Z, d)$, such that $Y_{1}$ and $Y_{2}$ are isometrically embedded in $(Z, d)$ and the Hausdorff distance between them is less than $2 \epsilon$ and $d_{Z}\left(p_{1}, p_{2}\right)<2 \epsilon$. If $\alpha \in G\left(Y_{1}, \delta_{1}, p_{1}\right)$, then it can be represented by some rectifiable closed curve $\gamma:[0,1] \rightarrow Y_{1}$. On $\Gamma:=\gamma([0,1])$ now choose points $x_{1}, \cdots, x_{m}, x_{i}=\gamma\left(t_{i}\right)$, with $x_{m}=x_{0}=p_{1}$ and $0=t_{0} \leq$ $t_{1} \leq \cdots \leq t_{m}=1$ such that for each $\gamma_{i}:=\left.\gamma\right|_{\left[t_{i}, t_{i+1}\right]}, i=0, \cdots, m-1$, one has $d\left(x_{i}, x_{i+1}\right) \leq L\left(\gamma_{i}\right)<5 \epsilon$. We will refer to this as a $5 \epsilon$-partition of $\gamma$. Since $d_{H}^{Z}\left(Y_{1}, Y_{2}\right) \leq 2 \epsilon$, for each $x_{i}, i=0, \cdots, m-1$, we can choose points $y_{i} \in Y_{2}$ satisfying $d_{Z}\left(x_{i}, y_{i}\right)<2 \epsilon$ and set $y_{m}:=y_{0}=p_{2}$. Connected $y_{i}$ to $y_{i+1}$ by a minimizing geodesic, $\bar{\gamma}_{i}, i=0, \cdots, m-1$ of length less than $9 \epsilon$. This yields a closed curve $\bar{\gamma}$ in $Y_{2}$, consisting of $m$ minimizing segments and having $p_{2}$ as its base point. Now define

$$
\Phi(\alpha)=\Phi([\gamma]):=[\bar{\gamma}] \in G\left(Y_{2}, \delta_{2}, p_{2}\right) .
$$

First we need to verify that $\Phi$ is well defined. Using the fact that $9 \epsilon<\delta_{1} / 2<$ $\delta_{2} / 2$, one easily sees that $[\bar{\gamma}]$ doesn't depend on the choice of minimizing geodesics $\bar{\gamma}_{i}$, nor on the choice of points $y_{i} \in Y_{2}$, nor on the special partition $\left\{x_{1}, \cdots, x_{m}\right\}$ of $\gamma([0,1])$. Moreover using additionally the uniform continuity of a homotopy one can see that $[\bar{\gamma}]$ only depends on the homotopy class of $\gamma$. It thus is also easy to check that $\Phi$ is a homomorphism from $\pi_{1}\left(Y_{1}, p_{1}\right)$ to $G\left(Y_{2}, \delta_{2}, p_{2}\right)$. However $\alpha \in G\left(Y_{1}, \delta_{1}, p_{1}\right)$ not $\pi_{1}\left(Y_{1}, p_{1}\right)$.

Suppose $\gamma_{1}$ and $\gamma_{2}$ are both representatives of $\alpha \in G\left(Y_{1}, \delta_{1}, p_{1}\right)$. Then $\gamma_{1} * \gamma_{2}^{-1}$ is homotopic to a loop $\gamma_{3}$ generated by loops of the form $(\alpha * \beta) * \alpha^{-1}$, where $\beta$ is a closed path lying in a ball of radius $\delta_{1}$ and $\alpha$ is a path from $p_{1}$ to $\beta(0)$. So $\left[\overline{\gamma_{1}}\right]=\left[\overline{\gamma_{3}}\right] *\left[\overline{\gamma_{2}}\right]$ and we need only show that $\left[\overline{\gamma_{3}}\right]$ is trivial in $G\left(Y_{2}, \delta_{2}, p_{2}\right)$.

In fact $\overline{\gamma_{3}}$ can be chosen as follows. The $y_{i}$ 's corresponding to the $x_{i}$ 's from the $\beta$ segments of $\gamma_{3}$ are all within $\delta_{1}+2 \epsilon$ of a common point and the minimal geodesics between them are within $\delta_{1}+(2+9 / 2) \epsilon<\delta_{2}$. Furthermore, the $y_{i}$ 's corresponding to the $x_{i}$ 's from the $\alpha$ and $\alpha^{-1}$ segments of the curve can be chosen to correspond. Thus $\overline{\gamma_{3}}$ is generated by loops of the form $(\alpha * \beta) * \alpha^{-1}$, where $\beta$ is a closed path lying in a ball of radius $\delta_{2}$ and $\alpha$ is a path from $p_{2}$ to $\beta(0)$. So it is trivial.

Last, we need to show that $\Phi$ is onto. If $\bar{\alpha} \in G\left(Y_{2}, \delta_{2}, p_{2}\right)$, it can be represented by some rectifiable closed curve $\sigma$. Choose an $\epsilon$-partition $\left\{y_{0}, \cdots, y_{m}\right\}$ of $\sigma$ such that for all $i=0, \cdots, m-1$, one has $L\left(\left.\sigma\right|_{\left[t_{i}, t_{i+1}\right]}\right)<\epsilon,\left(y_{i}=\sigma\left(t_{i}\right)\right)$. Now choose corresponding points $x_{i} \in Y_{1}$ satisfying $d\left(x_{i}, y_{i}\right)<2 \epsilon$ and we can connect those points by minimizing curves in $Y_{1}$. This yields a piecewise length minimizing $\gamma$ : $[0,1] \rightarrow Y_{1}$ with base point $x_{0}$, and because of $d\left(x_{i}, x_{i+1}\right)<5 \epsilon$ the curve $\gamma$ allows a $5 \epsilon$-partition and $[\gamma] \in G\left(Y_{1}, \delta_{1}, p_{1}\right)$. Now $\Phi([\gamma])=\bar{\alpha} \in G\left(Y_{2}, \delta_{2}, p_{2}\right)$ because $\Phi([\gamma])$ was shown above not to depend upon the choice of the $y_{i}$ as long as $d_{Z}\left(x_{i}, y_{i}\right)<2 \epsilon$ and the $x_{i}$ were a $5 \epsilon$-partition of a representative curve $\gamma$.

Therefore $\Phi$ is surjective.

Let $\left(M_{i}, p_{i}\right)$ be a sequence of connected locally path-connected spaces that converge to $(Y, p)$ in the pointed Gromov-Hausdorff topology. Denote by $\tilde{M}_{i}^{\delta}$ the $\delta$-covering of $\left(M_{i}, p_{i}\right), \pi_{1}\left(M_{i}, \delta, p_{i}\right)$ the covering group and

$$
G\left(M_{i}, \delta, p_{i}\right)=\pi_{1}\left(M_{i}, p_{i}\right) / \pi_{1}\left(M_{i}, \delta, p_{i}\right)
$$

the deck transformation on $\tilde{M}_{i}^{\delta}$.

Now Theorem 3.4 gives us the following. 
Corollary 3.5. If $\left(M_{i}, p_{i}\right)$ is a sequence of connected locally path-connected spaces with diam $\left(M_{i}\right) \leq D$ which converges to $(Y, p)$ in the pointed Gromov-Hausdorff topology, then for any $\delta_{1}<\delta_{2}$, there exists $N$ sufficiently large depending upon $\delta_{2}$ and $\delta_{1}$ such that $\forall i \geq N$ there is a surjective map $\Phi_{i}: G\left(M_{i}, \delta_{1}\right) \rightarrow G\left(Y, \delta_{2}\right)$.

Using this we can show that in the Gromov-Hausdorff limit the $\delta$-covering of the sequence converges to a cover which is almost the $\delta$-covering of the limit. The fact that it isn't quite the $\delta$-covering is seen by the following simple example. Take a sequence of flat tori of side lengths 1 by $(n-1) /(2 n)$. The $\delta=1 / 2$ cover of these tori are cylinders since all loops of length $<1 / 2$ are not unraveled. However, they converge to a torus of side lengths 1 by $1 / 2$ whose $\delta=1 / 2$ cover is Euclidean space, which is a cover of the limit cylinder. However, for any $\delta_{2}<\delta$, the $\delta_{2}$-cover of the limit is a cylinder.

Theorem 3.6. If $M_{i}$ with diam $\left(M_{i}\right) \leq D$ converges to $Y$ in the Gromov-Hausdorff metric and the $\delta$-covering of $M_{i},\left(\tilde{M}_{i}^{\delta}, \tilde{p}_{i}\right)$, converges in the pointed Gromov-Hausdorff metric to $\left(Y^{\delta}, \tilde{p}_{\infty}\right)$, then $\left(Y^{\delta}, \tilde{p}_{\infty}\right)$ is a covering space of $Y$, which is covered by the $\delta$-cover of $Y, \tilde{Y}^{\delta}$. Furthermore, for all $\delta_{2}>\delta, Y^{\delta}$ covers $\tilde{Y}^{\delta_{2}}$. So we have covering projections mapping

$$
\tilde{Y}^{\delta} \rightarrow Y^{\delta} \rightarrow \tilde{Y}^{\delta_{2}} \rightarrow Y
$$

Proof. Let $\pi_{i}^{\delta}: \tilde{M}_{i}^{\delta} \rightarrow M_{i}$ be the covering map. It is distance decreasing by construction. After possibly passing to a subsequence it follows from a generalized version of the Arzela-Ascoli theorem (see e.g. [Pe2, page 279, Lemma 1.8]) that $\pi_{i}^{\delta}: \tilde{M}_{i}^{\delta} \rightarrow M_{i}$ will converge to a distance decreasing map $\pi^{\delta}: Y^{\delta} \rightarrow Y$.

First, since $\tilde{M}_{i}^{\delta}$ is the $\delta$-covering space, the covering map $\pi_{i}^{\delta}: \tilde{M}_{i}^{\delta} \rightarrow M_{i}$ must be an isometry on any ball of radius $<\delta$. As $\pi_{i}^{\delta}$ converges to $\pi^{\delta}$ this property must be carried over to $\pi^{\delta}: Y^{\delta} \rightarrow Y$. Hence $\pi^{\delta}: Y^{\delta} \rightarrow Y$ is a covering map.

So we have three covering spaces of $Y, \tilde{Y}^{\delta}, Y^{\delta}$ and $\tilde{Y}^{\delta_{2}}$. By the Unique Lifting Theorem [Ma, Lemma 3.1, page 123] if $\tilde{Y}_{1}$ and $\tilde{Y}_{2}$ are covers of $Y$, then $\tilde{Y}_{1}$ covers $\tilde{Y}_{2}$ if every closed curve in $Y$ which lifts to a closed curve in $\tilde{Y}_{1}$ also lifts to a closed curve in $\tilde{Y}_{2}$.

Now if $C$ is a closed curve in $Y$ whose lift to $\tilde{Y}^{\delta}$ is closed, then it is homotopic to a curve consisting of paths, loops within $\delta$-balls and reverse paths. So its lift to $Y^{\delta}$ is also closed since $\pi^{\delta}$ is an isometry on $\delta$-balls. Therefore $\tilde{Y}^{\delta}$ covers $Y^{\delta}$.

If $\delta_{2}>\delta$, we want to show $Y^{\delta}$ covers $\tilde{Y}^{\delta_{2}}$. Suppose not. Then there is a closed curve $C$ in $Y$ whose lift to $Y^{\delta}$ is closed but whose lift to $\tilde{Y}^{\delta_{2}}$ is not a closed loop.

Since the lift of $C$ in $\tilde{Y}^{\delta_{2}}$ is not closed, $\Phi_{\delta_{2}}([C]) \in G\left(Y, \delta_{2}\right)$ is nontrivial. Using Corollary [3.5] we can find $N$ sufficiently large so that $\Phi_{i}: G\left(M_{i}, \delta\right) \rightarrow G\left(Y, \delta_{2}\right)$ is surjective. In particular we can find curves $C_{i}$ which converge to $C$ in the GromovHausdorff sense, such that $\Phi_{i}\left(\left[C_{i}\right]\right)=[C]$. Since, $\left[C_{i}\right]$ are nontrivial their lifts to $\tilde{M}_{i}^{\delta}$ run between points $\tilde{C}_{i}(0) \neq \tilde{C}_{i}(1)$. Furthermore, by Lemma 3.1,

$$
d_{\tilde{Y}_{i}^{\delta}}\left(\tilde{C}_{i}(0), \tilde{C}_{i}(1)\right) \geq \delta .
$$

In the limit, the lifted curves $\tilde{C}_{i}$ converge to the lift of the limit of the curves $\tilde{C}$ in $Y^{\delta}$ and

$$
d_{Y^{\delta}}(\tilde{C}(0), \tilde{C}(1)) \geq \delta .
$$

This implies that $\tilde{C}$ is not closed and we have a contradiction. 
Theorem 3.7. If there exists $\delta_{Y}$ such that for all $\delta<\delta_{Y}$ we have $\tilde{Y}^{\delta}=\tilde{Y}^{\delta_{Y}}$, then the universal cover of $Y$ exists and is $\tilde{Y}^{\delta_{Y}}$.

Proof. We need only show that given any cover, $\tilde{Y}^{\prime}$, of $Y, \tilde{Y}^{\delta_{Y}}$ covers $\tilde{Y}^{\prime}$. By Theorem $12 \mathrm{in}$ Sp, p. 81], $p: \tilde{Y}^{\prime} \rightarrow Y$ is a covering projection only if there is an open covering $\mathcal{U}$ of $Y$ and a point $\tilde{x} \in \tilde{Y}^{\prime}$ such that

$$
\pi(\mathcal{U}, p(\tilde{x})) \subset p_{\#} \pi\left(\tilde{Y}^{\prime}, \tilde{x}\right) .
$$

Since $Y$ is compact, there is a finite sub-cover, $\mathcal{U}^{\prime}$, such that $\pi(Y, \mathcal{U}, p(\tilde{x}))=$ $\pi\left(Y, \mathcal{U}^{\prime}, p(\tilde{x})\right)$. For all $y \in Y$ there is an $r_{y}>0$ such that $B\left(y, r_{y}\right) \subset U$ and $U \in \mathcal{U}^{\prime}$. Let

$$
\mathcal{V}=\left\{B_{y}\left(r_{y}\right): y \in Y\right\} .
$$

Now $\mathcal{V}$ also has a finite sub-cover $\mathcal{V}^{\prime}$, and taking

$$
\delta_{0}=\min \left\{r_{y}: B_{y}\left(r_{y}\right) \in \mathcal{V}^{\prime}, \delta_{Y}\right\},
$$

we have a $\delta_{0}$ open cover, $\mathcal{U}_{\delta_{0}}$, which refines $\mathcal{U}$. Thus by [Sp p. 81, st. 8],

$$
\pi\left(\mathcal{U}_{\delta_{0}}, p(\tilde{x})\right) \subset p_{\#} \pi\left(\tilde{Y}^{\prime}, \tilde{x}\right) .
$$

In particular, $\tilde{Y}^{\delta_{0}}$ covers $\tilde{Y}^{\prime}$. However $\delta_{0} \leq \delta_{Y}$, so $\tilde{Y}^{\delta_{Y}}=\tilde{Y}^{\delta_{0}}$ covers $\tilde{Y}^{\prime}$.

We now prove that the revised fundamental group of the limit space of a sequence of simply connected compact length spaces is trivial [Theorem 1.5. The example in Section 2 shows that this is as much as we can hope for.

Proof of Theorem 1.5. Since $M_{i}$ are simply connected, for all $\delta>0$, we know $G\left(M_{i}, \delta\right)$ is trivial. Thus by Corollary 3.5, we know that for all $\delta>0, G(Y, \delta)$ is trivial as well. Thus $\tilde{Y}^{\delta}=Y$ for all $\delta$ and we satisfy the conditions of Theorem 3.7 So the universal cover exists and has a trivial group of deck transformations.

We now apply these results to study limits of Riemannian manifolds with a lower bound on Ricci curvature.

\section{Ricci Curvature}

In this section we assume $Y$ is a Gromov-Hausdorff limit of compact manifolds $\left\{\left(M_{i}^{n}, p_{i}\right)\right\}$ satisfying (1.1), that is Ric $M_{i}^{n} \geq-(n-1)$ and $\operatorname{Diam}\left(M_{i}\right) \leq D$. Recall that, by the Gromov Precompactness Theorem [Gr, Thm. 5.3], a subsequence of any sequence of covering spaces of such manifolds converges in the Gromov-Hausdorff sense to some limit space. In particular, we know the following.

Lemma 4.1. If $Y$ is the limit of $\left\{\left(M_{i}^{n}, p_{i}\right)\right\}$ satisfying (1.1) and $\delta>0$, then there exists a subsequence such that $\left\{\left(\tilde{M}_{i_{j}}^{\delta}, \tilde{p}_{i}\right)\right\}$ converges in the Gromov-Hausdorff sense to some limit space $Y^{\delta}$ with all the properties of Theorem 3.6.

Thus we can apply Cheeger-Colding's result [ChCo $]$ to show that the BishopGromov volume comparison theorem holds on $Y^{\delta}$. Recall ChCo

Theorem 4.2 (Cheeger-Colding). Given any sequence of pointed manifolds, $\left\{\left(M_{i}^{n}\right.\right.$, $\left.\left.p_{i}\right)\right\}$, for which Ric $M_{i}^{n} \geq-(n-1)$ holds, there is a subsequence, $\left\{\left(M_{j}^{n}, p_{j}\right)\right\}$, convergent to some $\left(Y^{m}, y\right)$ in the pointed Gromov-Hausdorff sense, and there is a 
measure $\bar{V}_{\infty}$ on $Y$ satisfying Bishop-Gromov's volume comparison theorem, i.e. for $z \in Y^{m}, r_{1} \leq r_{2}$, the following holds:

$$
\frac{\bar{V}_{\infty}\left(z, r_{1}\right)}{\bar{V}_{\infty}\left(z, r_{2}\right)} \geq \frac{V_{n,-1}\left(r_{1}\right)}{V_{n,-1}\left(r_{2}\right)},
$$

where $V_{n,-1}\left(r_{1}\right)$ is the volume of a ball of radius $r_{1}$ in the simply connected space of dimension $n$ and curvature $\equiv-1$.

Therefore, we immediately have the following

Corollary 4.3. Bishop-Gromov's volume comparison theorem (4.11) holds on the Gromov-Hausdorff limit, $Y^{\delta}$, of any converging subsequence of $\delta$-coverings of $M_{i}$.

In $\mathrm{ChCo}$, Cheeger and Colding prove the following theorem about the regularity of the limit spaces of spaces with Ricci curvature curvature bounded below.

Definition 4.1. A regular point, $y$, in a limit space, $Y$, is a point such that there exists $k$ such that every tangent cone at $y$ is isometric to $\mathbb{R}^{k}$.

Theorem 4.4 (Cheeger-Colding). If $Y$ is the limit space of a sequence of $M_{i}^{n}$ with Ric $M_{i}^{n} \geq-(n-1)$, then the set of regular points has positive measure, $\bar{V}_{\infty}(\mathcal{R})>0$. In fact, the regular points are dense in $Y$.

Although we cannot control the topology in general, near a regular point, we can control the $\delta$-covers. In fact we can control these $\delta$-covers above points with poles in all their tangent cones. Recall that a tangent cone $Y^{\infty}$, has a pole at $y_{\infty}$ if $\forall x \in Y^{\infty}$, there is a length minimizing curve emanating from $y_{\infty}$ that passes through $x$ and extends minimally to $\infty$. Naturally this occurs at a regular point.

Theorem 4.5. Let $Y$ be the Gromov-Hausdorff limit of a sequence of compact manifolds such that

$$
\text { Ric } \geq-(n-1) K \text { where } n \geq 3, K>0 \text {. }
$$

If $y \in Y$ is a point such that there exists a tangent cone, $Y^{\infty}, y_{\infty}$, that has a pole at $y_{\infty}$, then there exists $r_{y}>0$, such that for all $\delta>0, B\left(y, r_{y}\right)$ lifts isometrically to $Y^{\delta}$.

The proof of this theorem uses an idea similar to one used in So. Here, however, we have an arbitrary lower bound on Ricci curvature and are concerned with eliminating small loops rather than large ones. In both cases we need to look at the Excess Theorem of Abresch and Gromoll from a new perspective AbGl. This new perspective is required because the original excess theorem has an inequality that includes the distance from a point to a minimal geodesic. Such a distance does not adapt well here because a curve of minimal length in a limit space is not necessarily the limit of minimal geodesics. Thus we begin with the following lemma.

Lemma 4.6. Let $M^{n}$ be a complete Riemannian manifold satisfying [4.12). There exists a constant

$$
S=S_{n, K}=\min \left\{\frac{1}{8}, \frac{1}{4 \cdot 3^{n}} \frac{1}{\cosh (\sqrt{K} / 4)} \frac{n}{n-1}\left(\frac{n-2}{n-1}\right)^{n-1}\left(\frac{\sqrt{K}}{\sinh \sqrt{K}}\right)^{n-1}\right\}
$$

such that if $\gamma$ is a minimal geodesic of length $D \leq 1$ and $x \in M^{n}$ satisfying

$$
d(x, \gamma(0)) \geq\left(S_{n, K}+1 / 2\right) D \text { and } d(x, \gamma(D)) \geq\left(S_{n, K}+1 / 2\right) D
$$




$$
d(x, \gamma(D / 2)) \geq 3 S_{n, K} D .
$$

Note that in the case with nonnegative Ricci curvature, $D$ can have arbitrary length and $S$ has no hyperbolic terms [So].

Proof. First we recall the Excess Theorem [AbG1, Prop. 2.3]. If $r_{0}=d(x, \gamma(0))$, $r_{1}=d(x, \gamma(D))$ and $l=d(x, \gamma)<\min \left\{r_{0}, r_{1}\right\}$, then

$$
e(x)=r_{0}+r_{1}-D \leq 2\left(\frac{n-1}{n-2}\right)\left(\frac{1}{2} C_{3} l^{n}\right)^{1 /(n-1)},
$$

where

$$
C_{3}=\frac{n-1}{n}\left(\frac{\sinh \sqrt{K} l}{\sqrt{K} l}\right)^{n-1} \sqrt{K}\left[\operatorname{coth} \sqrt{K}\left(r_{0}-l\right)+\operatorname{coth} \sqrt{K}\left(r_{1}-l\right)\right] .
$$

Now suppose $l>\min \left\{r_{0}, r_{1}\right\}$, then

$$
d(x, \gamma(D / 2)) \geq l \geq(S+1 / 2) D \geq 3 S D .
$$

So we need only consider the case where $l<\min \left\{r_{0}, r_{1}\right\}$ and we can apply (4.15).

Let us assume on the contrary that (4.14) does not hold. Then $l<3 S D$, $r_{0}-l>(1 / 2+S) D-3 S D>D / 4$ and similarly $r_{1}-l>D / 4$. Therefore

$$
\begin{aligned}
e(x)<2\left(\frac{n-1}{n-2}\right)\left[\frac{1}{2}\left(\frac{n-1}{n}\right)\left(\frac{\sinh (\sqrt{K} 3 S D)}{\sqrt{K} 3 S D}\right)^{n-1}\right. & \left.\cdot 2 \sqrt{K} \operatorname{coth}\left(\sqrt{K} \frac{D}{4}\right)(3 S D)^{n}\right]^{1 /(n-1)} \\
\leq & 2\left(\frac{n-1}{n-2}\right)\left[\frac{1}{2}\left(\frac{n-1}{n}\right)\left(\frac{\sinh \sqrt{K}}{\sqrt{K}}\right)^{n-1}\right. \\
& \left.\cdot 2 \sqrt{K} \frac{\cosh (\sqrt{K} / 4)}{\sqrt{K} \frac{D}{4}}(3 S D)^{n}\right]^{1 /(n-1)} \\
\leq & 2 D\left(\frac{n-1}{n-2}\right)\left[4\left(\frac{n-1}{n}\right)\left(\frac{\sinh \sqrt{K}}{\sqrt{K}}\right)^{n-1} \cosh (\sqrt{K} / 4)(3 S)^{n}\right]^{1 /(n-1)} .
\end{aligned}
$$

On the other hand, $e(x)=r_{0}+r_{1}-D \geq 2(S+1 / 2) D-D=2 S D$, so

$$
S<\frac{n-1}{n-2}\left[4\left(\frac{n-1}{n}\right)\left(\frac{\sinh \sqrt{K}}{\sqrt{K}}\right)^{n-1} \cosh (\sqrt{K} / 4)(3 S)^{n}\right]^{1 /(n-1)} .
$$

This gives

$$
S>4^{-1} 3^{-n} \frac{1}{\cosh (\sqrt{K} / 4)} \frac{n}{n-1}\left(\frac{n-2}{n-1}\right)^{n-1}\left(\frac{\sqrt{K}}{\sinh \sqrt{K}}\right)^{n-1},
$$

contradicting (4.13).

We will now apply this lemma to prove Theorem 4.5 . 
Proof of Theorem 4.5. Assume on the contrary that for all $r>0$ there is a $\delta_{r}>0$ such that the ball $B(y, r)$ does not lift isometrically to $Y^{\delta_{r}}$. Let $G^{\delta}$ denote the deck transformation group on $Y^{\delta}$. Thus, there exist $r_{i} \rightarrow 0, \delta_{i}=\delta_{r_{i}}$, and $g_{i} \in G^{\delta_{i}}$ such that $d_{i}=d_{Y^{\delta_{i}}}\left(\tilde{y}, g_{i} \tilde{y}\right) \in\left(0,2 r_{i}\right) \subset(0,1]$. In fact, we can choose $g_{i}$ so that

$$
d_{Y^{\delta_{i}}}\left(\tilde{y}, g_{i} \tilde{y}\right) \leq d_{Y^{\delta_{i}}}(\tilde{y}, h \tilde{y}) \quad \forall h \in G^{\delta_{i}} .
$$

Next we will find a length minimizing curve, $\tilde{C}_{i}$, running from $\tilde{y}$ to $g_{i} \tilde{y}$ which has the property that it passes through a particular point $\tilde{z}_{i}=\tilde{C}_{i}\left(d_{i} / 2\right)$ which is the limit of halfway points of length minimizing curves in the sequence $\tilde{M}_{j}{ }^{\delta_{i}}$. We do this so that we can apply Lemma 4.6 to $M_{j}$.

To construct $\tilde{C}_{i}$, we first let $\tilde{y}_{j}$ and $\tilde{y}_{j}^{i}$ be points in $\tilde{M}_{j}{ }^{\delta_{i}}$ which are close to $\tilde{y}$ and $g_{i} \tilde{y}$. So $d_{\tilde{M}_{j}}\left(\tilde{y}_{j}, \tilde{y}_{j}^{i}\right)=d_{i, j}$ converges to $d_{i}$. Let $\tilde{z}_{j}^{i}$ be midpoints of minimal geodesics $\gamma_{j}^{i}$, running from $\tilde{y}_{j}$ to $\tilde{y}_{j}^{i}$. Taking a subsequence of $j \rightarrow \infty$, there is a point $\tilde{z}_{i} \in Y^{\delta_{i}}$ which is halfway between $\tilde{y}$ to $g_{i} \tilde{y}$. Let $\tilde{C}_{i}$ be a length minimizing curve running from $\tilde{y}$ to $\tilde{z}_{i}$ and then to $g_{i} \tilde{y}$. Finally let $C_{i}$ be the projection of $\tilde{C}_{i}$ to $Y$.

Now, imitating the proof of the Halfway Lemma of [So, and using (4.17), we know $C_{i} \in Y$ is minimizing halfway around, $d_{Y}\left(C_{i}(0), C_{i}\left(d_{i} / 2\right)\right)=d_{i} / 2$.

We choose a subsequence of these $i$ such that $(Y, y)$ rescaled by $d_{i}$ converges to a tangent cone $\left(Y^{\infty}, y_{\infty}\right)$. So

$$
d_{G H}\left(B\left(y, 10 d_{i}\right) \subset Y, B\left(y_{\infty}, 10 d_{i}\right)\right)<\epsilon_{i} d_{i},
$$

where $\epsilon_{i}$ converges to 0 .

Let $S$ be the constant from Lemma 4.6 Since $Y^{\infty}$ has a pole at $y_{\infty}$, we know there is a length minimizing curve running from $y_{\infty}$ through any point in $\partial B\left(y_{\infty}, d_{i} / 2\right)$ to $\partial B\left(y_{\infty}, d_{i} / 2+2 S d_{i}\right)$. Thus by (4.18),

$$
\forall x \in \partial B\left(y, d_{i} / 2+2 S d_{i}\right) \subset Y,
$$

we have points

$$
x_{\infty} \in A n n_{y_{\infty}}\left(d_{i} / 2+2 S d_{i}-\epsilon_{i} d_{i}, d_{i} / 2+2 S d_{i}+\epsilon_{i} d_{i}\right)
$$

and

$$
y_{i} \in A n n_{y_{\infty}}\left(d_{i} / 2-\epsilon_{i} d_{i}, d_{i} / 2+\epsilon_{i} d_{i}\right)
$$

such that

$$
\begin{aligned}
d_{Y}\left(x, C_{i}\left(d_{i} / 2\right)\right) & <d_{Y^{\infty}}\left(x_{\infty}, y_{i}\right)+\epsilon_{i} d_{i}, \\
& \leq 2 \epsilon_{i} d_{i}+2 S d_{i}+\epsilon_{i} d_{i} .
\end{aligned}
$$

Now we will imitate the Uniform Cut Lemma of [So], to show that for all $x \in$ $\partial B\left(y, d_{i} / 2+2 S d_{i}\right)$, we have $l_{i}=d_{Y}\left(x, C_{i}\left(d_{i} / 2\right)\right) \geq(3 S) d_{i}$. This will provide a contradiction for $\epsilon_{i}<S / 2$ and we are done.

First we lift our points $x$ and $y$ to the cover $\tilde{Y}^{\delta_{i}}$ as follows. We lift $y$ to the point $\tilde{y}$ and we lift the closed loop $C_{i}$ to the curve $\tilde{C}_{i}$ running from $\tilde{y}$ through $z_{i}=C_{i}\left(\tilde{d}_{i} / 2\right)$ to $g_{i} \tilde{y}$. Then if $\sigma$ is a length minimizing curve of length $l_{i}$ running from $C_{i}\left(d_{i} / 2\right)$ to $x$, we lift it to $\tilde{Y}^{\delta_{i}}$ so it runs from $\tilde{z}_{i}$ to a new point, $\tilde{x}$. Note that by our choice of $x$ in (4.19),

$$
d_{\tilde{Y} \delta_{i}}\left(g_{i} \tilde{y}, \tilde{x}\right) \geq d_{Y}(y, x)=d_{i} / 2+2 S d_{i}
$$

and so is $d_{\tilde{Y} \delta_{i}}(\tilde{y}, \tilde{x})$. 
By our choice of $\tilde{C}_{i}$ and $\tilde{z}_{i}$, we know there are corresponding points in $\tilde{M}_{j}^{\delta_{i}}$. That is there is a triangle formed by $\tilde{y}_{j}, \tilde{y}_{j}^{i}$, with a minimal geodesic $\gamma_{j}^{i}$ running between them and some point $\tilde{x}_{j}$ such that

$$
\begin{aligned}
d_{i, j}=d_{\tilde{M}_{j} \delta_{i}}\left(\tilde{y}_{j}, \tilde{y}_{j}^{i}\right) & \rightarrow d_{i}, \\
d_{\tilde{M}_{j}^{\delta_{i}}}\left(\tilde{y}_{j}, \tilde{x}_{j}\right) & \rightarrow d_{\tilde{Y}^{\delta_{i}}}(\tilde{y}, \tilde{x})=(1 / 2+2 S) d_{i}, \\
d_{\tilde{M}_{j}^{\delta_{i}}}\left(\tilde{y}_{j}^{i}, \tilde{x}_{j}\right) & \rightarrow d_{\tilde{Y}^{\delta_{i}}}\left(g_{i} \tilde{y}, \tilde{x}\right)=(1 / 2+2 S) d_{i}, \\
l_{i, j}=d_{\tilde{M}_{j} \delta_{i}}\left(\tilde{\gamma}_{j}^{i}\left(d_{i, j} / 2\right), \tilde{x}_{j}\right) & \rightarrow d_{\tilde{Y}^{\delta_{i}}}\left(\tilde{z}_{i}, \tilde{x}\right)=l_{i} .
\end{aligned}
$$

So for $j$ sufficiently large, we have

$$
d_{\tilde{M}_{j} \delta_{i}}\left(\tilde{y}_{j}, \tilde{x}_{j}\right) \geq(1 / 2+S) d_{i, j} \text { and } d_{\tilde{M}_{j} \delta_{i}}\left(\tilde{y}_{j}^{i}, \tilde{x}_{j}\right) \geq(1 / 2+S) d_{i, j}
$$

and can apply Lemma 4.6 to get

$$
l_{i, j} \geq 3 S d_{i, j}
$$

Taking $j$ to infinity, we get the limit of this bound in $\tilde{Y}^{\delta_{i}}$, namely $l_{i} \geq 3 S d_{i}$. This contradicts (4.22) for $\epsilon_{i}<S / 2$ and we are done.

Note 4.7. In the non-collapsed case, namely when the sequence of compact manifolds $M_{i}^{n}$ satisfy (1.3), every tangent cone of the limit space at every point is polar ChCo. By Theorem 4.5 for every $y \in Y$, there exists $r_{y}>0$, such that for all $\delta>0, B\left(y, r_{y}\right)$ lifts isometrically to $Y^{\delta}$ for all $\delta>0$. Since $Y$ is compact, this implies that the $Y^{\delta}$ stabilize, i.e. there exists $\delta_{Y}$ depending on $Y$ such that for all $\delta<\delta_{Y}$, we have $Y^{\delta}=Y^{\delta_{Y}}$.

Using Theorem 4.5 and volume comparison we will prove that this is also true in the collapsed case.

Theorem 4.8. There exists $\delta_{Y}$ depending on $Y$ such that for all $\delta<\delta_{Y}$, we have $Y^{\delta}=Y^{\delta_{Y}}$ and $G^{\delta}=G^{\delta_{Y}}$. Therefore $\tilde{Y}^{\delta}$ are also same for all small $\delta>0$.

Proof. Note that from Theorem 3.6 if $Y^{\delta}$ do not stabilize for $\delta$ small, then neither do the $\delta$-covers $\tilde{Y}^{\delta}$. So there exists a sequence of $\delta_{i}>0$ with $\delta_{1} \leq D, \delta_{i}>10 \delta_{i+1}$ such that all $\tilde{Y}^{\delta_{i}}$ and $G\left(Y, \delta_{i}\right)$ are distinct. In particular there are elements of $G\left(Y, \delta_{i}\right)$ which are trivial in $G\left(Y, \delta_{i-1}\right)$. So there exist $q_{i} \in Y$, such that the $B_{q_{i}}\left(\delta_{i-1}\right)$ contains a noncontractible loop, $C_{i}$, which lifts non-trivially in $Y^{\delta_{i}}$. Since $C_{i}$ must lift to a union of balls $B_{g \tilde{q}_{i}}\left(\delta_{i-1}\right)$ in $\tilde{Y}^{\delta_{i}}$, there exists $g_{i}$ non-trivial in $G\left(Y, \delta_{i}\right)$ such that

$$
d_{\tilde{Y}_{i}^{\delta}}\left(g_{i} \tilde{q}_{i}, \tilde{q}_{i}\right)<2 \delta_{i-1} .
$$

So if $\alpha_{i} \subset Y$ is the projection of the minimal geodesic from $g_{i} \tilde{q}_{i}$ to $\tilde{q}_{i}$ it represents an element $g_{i}$ of $\pi_{1}(Y)$ which is mapped non-trivially into $G_{\delta_{i}}$ and trivially into $G_{2 \delta_{i-1}}$.

For any $j$, the limit cover $Y^{\delta_{j}}$ covers $Y^{\delta_{i}}$ for $i=1 \ldots j-1$. So $g_{1} \ldots g_{j-1}$ are distinct non-trivial deck transforms of $Y^{\delta_{j}}$. Furthermore, for any $q \in Y$, letting $\tilde{q}_{i}$ be the lift of $q_{i}$ closest to $\tilde{q} \in Y^{\delta_{j}}$, we have

$$
\begin{aligned}
d_{Y^{\delta_{j}}}\left(\tilde{q}, g_{i} \tilde{q}\right) & \leq d_{Y^{\delta_{j}}}\left(\tilde{q}, \tilde{q}_{i}\right)+d_{Y^{\delta_{j}}}\left(\tilde{q}_{i}, g_{i} \tilde{q}_{i}\right)+d_{Y^{\delta_{j}}}\left(g_{i} \tilde{q}_{i}, g_{i} \tilde{q}\right) \\
& \leq D+2 L\left(\alpha_{i}\right)+D \leq 4 D .
\end{aligned}
$$


Therefore we have for any $j$, there are $j-1$ distinct elements in $G^{\delta_{j}}$ with $l\left(g_{i}, \delta_{j}\right) \leq$ $4 D$.

On the other hand the total number of elements in $G^{\delta}$ of $\delta$-length $\leq 4 D$ is uniformly bounded for all $\delta$ in terms of geometry and topology of $Y$. To show this let us look at the lift of a regular point $p \in Y$ in the cover $Y^{\delta}$. We know by Theorem 4.5 there is a $\delta_{0}>0$ such that the ball of radius $\delta_{0}$ about $p$ is isometrically lifted to disjoint balls of radius $\delta_{0}$ in $Y^{\delta}$. Let $N$ be the number of distinct elements in $G^{\delta}$ of $\delta$-length $\leq 4 D$. Note that $g B\left(\tilde{p}, \delta_{0}\right)$ is contained in $B\left(\tilde{p}, 4 D+\delta_{0}\right)$ for all $g \in G^{\delta}$ with $l(g, \delta) \leq 4 D$. Thus applying Corollary 4.3 we have

$$
N \leq \frac{\bar{V}_{\infty}\left(\tilde{p}, 4 D+\delta_{0}\right)}{\bar{V}_{\infty}\left(\tilde{p}, \delta_{0}\right)} \leq \frac{V_{n,-1}\left(4 D+\delta_{0}\right)}{V_{n,-1}\left(\delta_{0}\right)} .
$$

This is a contradiction.

Note that $\delta_{Y}$ can not be uniformly bounded regardless of $Y$, because we only bound the number of elements of a certain length. Furthermore, the $\delta_{0}$ depend on the properties of the regular point in $Y$. Finally, even with a uniform lower bound on volume, a uniform bound on $\delta_{Y}$ would contradict Otsu's examples.

This result has several nice consequences. First, combining this with Theorem 3.7, we get the following.

Theorem 4.9. If $Y$ is the limit of a sequence of compact manifolds with uniformly bounded diameter and a uniform lower bound on Ricci curvature, then the universal covering space of $Y$ exists.

Note 4.10. In fact $Y^{\delta_{Y}}$ is the universal cover. It is unknown whether the universal cover is simply connected.

This theorem allows us to define the revised fundamental group of $Y, \bar{\pi}_{1}(Y)$ [Definition 2.3. We can now prove Theorem 1.1 which was stated in the introduction.

Proof of Theorem 1.1. First, there is always a surjection

$$
\pi_{1}\left(M_{i}, p\right) \rightarrow G\left(M_{i}, \delta\right)=\pi_{1}\left(M_{i}, p\right) / \pi_{1}\left(M_{i}, \delta, p\right) .
$$

Take $\delta=\delta_{Y} / 2$. Then by Theorem 3.4 there exists $N$ sufficiently large so that there is a surjection $\Phi_{i}: G\left(M_{i}, \delta\right) \rightarrow G\left(Y, \delta_{Y}\right)$. Since $\tilde{Y}^{\delta}$ is the universal cover of $Y, G\left(Y, \delta_{Y}\right)=\bar{\pi}_{1}(Y)$.

Theorems 4.9 and 3.6 imply the following.

Corollary 4.11. The universal cover of a limit space, $Y$, of compact manifolds, $M_{i}$, satisfying (1.1), is the limit of $\delta_{Y} / 2$-covers of a subsequence of the $M_{i}$.

Corollary 4.12. For all $\delta<\delta_{Y}$, there exists $N$ sufficiently large depending on $\delta$ and $\delta_{Y}$ such that $G\left(M_{i}, \delta\right)=\bar{\pi}_{1}(Y)$ for all $i \geq N$.

Proof. Choose $N$ sufficiently large so that

$$
d_{G H}\left(M_{i}, Y\right)<\min \left\{\frac{\delta}{20}, \frac{\delta_{Y}-\delta}{20}\right\} \quad \forall i \geq N .
$$

Then by Theorem [3.4, there exists a surjection from $G\left(M_{i}, \delta\right)$ to $G\left(Y, \delta_{Y}\right)$ and there is also a surjection from $G(Y, \delta / 2)=G\left(Y, \delta_{Y}\right)$ to $G\left(M_{i}, \delta\right)$. It is clear from the definition of these surjections in the proof that they commute. 
In the non-collapsed case, i.e. the sequence of compact manifolds $M_{i}^{n}$ satisfy (1.3), we can now prove Theorem 1.4 that the fundamental groups of $M_{i}$ are eventually isomorphic up to a finite group.

Proof of Theorem 1.4. This essentially follows from Corollary 4.12 and Anderson's estimate on the order of subgroups generated by short loops [An, page 268] (see also [Pe2, page 256]). Anderson's estimate states that there exist $L=L(n, H, V, D)$ and $N=N(n, H, V, D)$ such that if $M^{n}$ is a compact manifold satisfying (1.3), then any subgroup of $\pi_{1}(M)$ that is generated by loops of length $\leq L$ must have order $\leq N$. Now choose $\delta$ such that $\delta \leq \min \left\{L / 2, \delta_{Y} / 2\right\}$. Since the covering group of the $\delta$-cover of each $M_{i}, \pi_{1}\left(M_{i}, \delta\right)$, is generated by loops of length $\leq 2 \delta \leq L$, the order of $\pi_{1}\left(M_{i}, \delta\right)$ is uniformly bounded by $N(n, v, D)$. On the other hand, by Corollary 4.12, the deck transformation group of the $\delta$-covering space of $M_{i}$, $G\left(M_{i}, \delta\right)=\pi_{1}\left(M_{i}\right) / \pi_{1}\left(M_{i}, \delta\right)$, is isomorphic to $\bar{\pi}_{1}(Y)$ for all $i \geq i_{0}$. Setting $F_{i}=$ $\pi_{1}\left(M_{i}, \delta\right)$ finishes the proof.

\section{REFERENCES}

[AbGl] U. Abresch, D. Gromoll, On complete manifolds with nonnegative Ricci curvature, J. Amer. Math. Soc. 3 (1990) 355-374. MR 91a:53071

[An] M. Anderson, Short geodesics and gravitational instantons, J. Differential Geom. 31 (1990), 265-275. MR 91b:53040

[Ca] M. Cassorla, Approximating compact inner metric spaces by surfaces, Indiana Univ. Math. J. 41 (1992) 505-513. MR 93i:53042

[ChCo] J. Cheeger, T. Colding, On the structure of spaces with Ricci curvature bounded below I, J. Diff. Geom. 46 (1997) 406-480. MR 98k:53044

[Co] T. Colding, Ricci curvature and volume convergence, Ann. of Math. (2) 145 (1997), no. 3, 477-501. MR 98d:53050

[Gr] M. Gromov, Metric structures for Riemannian and non-Riemannian spaces, Progress in Math. 152, Birkhäuser, 1999. MR 2000d:53065

[Ma] W. Massey, A basic course in algebraic topology, GTM 127, Springer-Verlag, 1991. MR 92e:55001

[Me] X. Menguy, Examples with bounded diameter growth and infinite topological type. Duke Math. J. 102 (2000), no. 3, 403-412. MR 2001e:53041

[Ot] Y. Otsu, On manifolds of positive Ricci curvature with large diameter, Math. Z. 206 (1991) 255-264. MR 91m:53033

[Pl] G. Perelman, A. D. Aleksandrov spaces with curvatures bounded below. Part II, preprint.

[Pe1] P. Petersen, The fundamental group of almost non-negatively curved manifolds, 1989, unpublished.

[Pe2] P. Petersen, Riemannian geometry, GTM 171, Springer-Verlag, 1998. MR 98m:53001

[Ri] W. Rinow, Die innere Geometric der metrischen Raume, Springer, 1961. MR 23:A1290

[So] C. Sormani, Nonnegative Ricci curvature, small linear diameter growth and finite generation of fundamental groups,

[Sp] E. Spanier, Algebraic Topology, McGraw-Hill, Inc., 1966. MR 83i:55001

[Tu] W. Tuschmann, Hausdorff convergence and the fundamental group, Math. Z. 218 (1995) 207-211. MR 96c:53066

[Zh] S-H Zhu, A finiteness theorem for Ricci curvature in dimension three. J. Differential Geom. 37 (1993), no. 3, 711-727. MR 94f:53071

Department of Mathematics and Computer Science, Lehman College, City University of New York, Bronx, New York 10468

E-mail address: sormani@g230.lehman.cuny.edu

Department of Mathematics, University of California, Santa Barbara, California 93106

E-mail address: wei@math.ucsb.edu 